\title{
Detailed measurements of equivalence ratio modulations in premixed flames using laser Rayleigh scattering and absorption spectroscopy
}

\author{
Heiner Schwarz, Laurent Zimmer, Daniel Durox and Sébastien Candel
}

September 6, 2010

\author{
Laboratoire EM2C - UPR 288 - CNRS - ECP \\ 92295 Châtenay-Malabry, FRANCE \\ Tel.: (+33) 141131066 \\ Fax: $(+33) 147028035$ \\ Laurent.Zimmer@em2c.ecp.fr
}

\section{Accepted in Experiments in Fluids \\ DOI: $10.1007 /$ s00348-010-0972-3}

\begin{abstract}
Equivalence ratio non-uniformities may give rise to some of the instabilities observed in modern lean premixed combustion systems. The present work intends to investigate the influence of equivalence ratio perturbations on the dynamics of premixed flames. A burner equipped with a secondary injection system is used to generate equivalence ratio perturbations which are convected by the flow and impinge on a conical flame. Two laser-diagnostics, based on Rayleigh scattering and hydrocarbon infrared absorption respectively, are employed to give insight into the spatial and temporal evolution of the mixture composition field. Rayleigh scattering images also reveal the flame front dynamics providing an indication on the response of a weakly turbulent flame subject to mixture composition inhomogeneities. Laser light absorption provides a time resolved signal which is used to estimate the equivalence ration perturbation level. A theoretical model based on the G-equation is used to interpret the experimental data and compare the relative effects of velocity and equivalence ratio perturbations.
\end{abstract}

Keywords: Flame dynamics Rayleigh scattering Absorption spectroscopy Equivalence ratio perturbations 


\section{Introduction}

Much of the current research in combustion has been focused on methods allowing reductions in pollutant emissions and has concerned more specifically $\mathrm{NO}_{x}$ emissions. Lean premixed combustion systems are now widely used to this purpose in modern gas turbines because they yield considerably lower levels of $\mathrm{NO}_{x}$ than standard systems. Their development is however hindered by combustion dynamics phenomena which give rise to instabilities. Self-sustained oscillations are generally caused by a resonant coupling between the heat release rate fluctuation $q^{\prime}$ and pressure oscillations $p^{\prime}$. This can lead to severe engine damage or even destruction in extreme cases. An early analysis of this phenomenon is due to (Rayleigh, 1878) who concluded that instability required that pressure and heat release fluctuations be in phase. In mathematical form, the Rayleigh criterion indicates that a necessary condition for instability is that

$$
\int_{[T]} p^{\prime} q^{\prime} d t>0 .
$$

It can also be shown that the rate of change of nonsteady heat release constitutes a source term in the acoustic wave equation, and that amplification of acoustic energy is obtained if the fluctuations of pressure and heat release are in phase and if the corresponding source term exceeds damping (Schuller, 2003).

In premixed situations, the fundamental mechanisms giving rise to combustion dynamics may be represented schematically as in Fig. 1. Two driving processes are generally considered to start the coupling loop: velocity perturbations $v^{\prime}$ and equivalence ratio fluctuations $\phi^{\prime}$, like (Crocco and Cheng, 1956; Putnam, 1971; Lieuwen and Zinn, 1998; Candel, 2002). In the first case flame dynamics is governed by velocity fluctuations associated with system acoustic modes, whereas in the latter combustion is influenced by equivalence ratio oscillations induced by the differential response of the injection system subject to pressure perturbations.

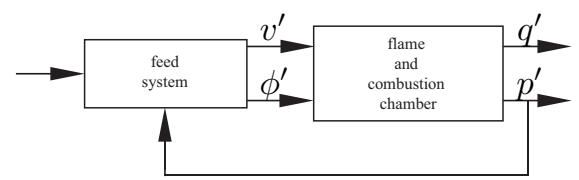

Figure 1: A flame can be subject to either velocity perturbations $v^{\prime}$ or fluctuations in equivalence ratio $\phi^{\prime}$ giving rise to heat release $q^{\prime}$ and pressure fluctuation $p^{\prime}$.

Much theoretical and experimental research has been carried out to investigate the former driving mechanism. It has been possible to describe the evolution of the flame front submitted to velocity perturbations and determine the corresponding heat release fluctuations induced by the changes in flame surface area. This is exemplified in a conical flame situation using a G-equation modeling approach as described in (Schuller et al., 2002). The predicted flame front 
wrinkling and the flame transfer function are in good agreement with experimental data.

The impact of equivalence ratio perturbations are of particular interest for lean premixed combustion processes where the burning velocity gradient

$$
\frac{1}{s_{L}} \frac{\partial s_{L}}{\partial \phi}
$$

plays a major role. Despite this fact, as far as flames subject to incident mixture fluctuations are concerned less experimental and theoretical data is at hand. Fluctuations and consequences in a combustion test rig at a full-scale industrial burner were shown in Biagioli et al. (2008). More detailed measurements on gas turbine models have been reported (Mongia et al. (1998), Lee et al. (2000) and subsequent papers, Zimmer and Tachibana (2007)) using different measurement techniques. However these data were either based on values obtained by averaging over the line of sight or on measurements over a small volume, without information on the gradients and spatial distribution of equivalence ratio. On the other hand, Meier et al. (2007) report phase-resolved equivalence ratio fluctuation measurements using Raman scattering on a model of gas turbine and show radial profiles of the induced perturbations. This indicates that in standard conditions, one cannot consider that equivalence ratio distribution is a plane wave. Furthermore, this indicates that line of sight absorption measurements will be influenced by the spatial distribution of the equivalence ratio and may only give an estimate of the local fluctuations.

Even though the online monitoring of equivalence ratio has been carried out in a variety of practical cases one also deduces from the results reported that well-controlled cases are still lacking. Ratner et al. (2002) are able to induce equivalence ratio fluctuations but the space time structure of these perturbations is not fully characterized. The recent experiments of Ax et al. (2009) concern mixture fraction oscillations in a low pressure environment (69 mbar), conditions rarely met in industrial applications. Kang et al. (2009) describe measurements with a turbulent flame in a fairly complex configuration and their results are difficult to interpret. On the computational side, unsteady simulations for a perturbed laminar flame have been recently reported by Birbaud (2006) and Birbaud et al. (2008) and some of the results for conical flames are presented in Fig. 2. Modeling of the transfer function of flames submitted to equivalence ratio modulations is reported by Sattelmayer (2003); Cho and Lieuwen (2005).

The objective of the present work is the investigation of the flame dynamics under incident equivalence ratio fluctuations. The aim is to develop an experimental setup where mixture properties and spatial distributions are well controlled and characterized. On the one hand this involves the design of a burner system equipped with means to impose equivalence ratio gradients with a quasi-plasne wave distribution, on the other hand a diagnostics setup that allows for spatial and temporal characterization of the inhomogeneities.

The next section describes the experimental setup of the diagnostics, namely planar Rayleigh scattering and absorption spectroscopy. The burner design is 


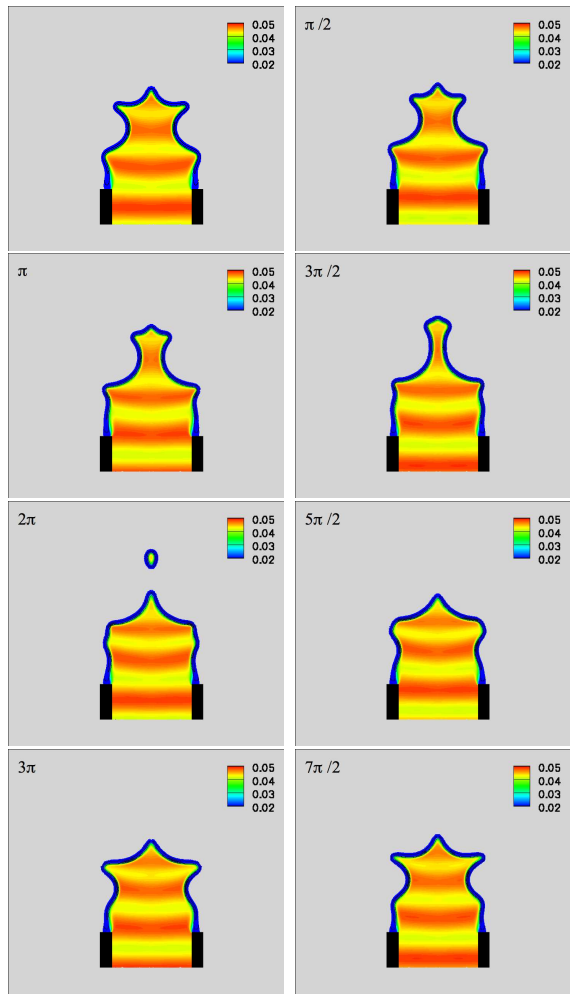

Figure 2: Numerical results of a direct simulation of flame dynamics under the influence of modulated mixture composition. The two columns depict the phase-evolution of the flame front and fuel mass fraction for an initially conical flame, excitation frequency $f=175 \mathrm{~Hz}$, modulation amplitude $\phi^{\prime} / \bar{\phi}=0.07$, mean velocity $\bar{v}=1 \mathrm{~m} / \mathrm{s}$ and $\bar{\phi}=0.8$. It is interesting to note that the flame responds at a subharmonic $f / 2$ of the modulation frequency. (originating from Birbaud (2006).)

described in the third section and the method for imposing fuel gradients on the flow is explained. Results for a weakly turbulent flame subject to equivalence ratio perturbations are reported in section 4 . The final section introduces a flame dynamics model relying on the G-equation which is then used to estimate the respective effects of velocity and equivalence ratio perturbations and interpret the experimental data. 


\section{Diagnostics}

\subsection{Planar Rayleigh scattering}

Rayleigh scattering designates the elastic scattering of electromagnetic radiation by molecules having a size inferior to the incident radiation wavelength. The scattered power (per solid angle $\Omega$ ) $I_{\Omega}=d P / d \Omega$ is essentially given by

$$
I_{\Omega}=I_{\mathrm{inc}} n \sigma_{\mathrm{eff}},
$$

where $I_{\text {inc }}$ is the incident field irradiance, $n$ the total number density and $\sigma_{\text {eff }}$ the effective Rayleigh cross-section. In a gas mixture, characterized by mole fractions $X_{i}$, the effective cross section $\sigma_{\text {eff }}$ is given by the mole fraction weighted average of the individual cross sections $\sigma_{i}$,

$$
\sigma_{\mathrm{eff}}=\sum_{i} \sigma_{i} X_{i}
$$

The two major applications of Rayleigh scattering in combustion science are the measurement of temperature and molar fractions. Scattering is proportional to number density $n$ and can be linked to gas temperature $T$ and pressure $p$ by means of the ideal gas law

$$
p=n k T \text {. }
$$

Here $k$ is the Boltzmann constant. In low velocity flows it is reasonable to assume that combustion takes place at constant pressure $p$, which leaves the two unknowns temperature $T$ and gas composition $X_{i}=n_{i} / n$.

\subsubsection{Rayleigh scattering for concentration determination}

In isothermal conditions, such as in a non-reacting, adiabatic flows, temperature $T$ is constant and Rayleigh scattering can provide information on gas composition. For a binary gas mixture comprising a hydrocarbon fuel $X_{\text {fuel }}$ and air $X_{\text {air }}=X_{\mathrm{N}_{2}}+X_{\mathrm{O}_{2}}$ according to equation (4) the effective cross-section is given by

$$
\sigma_{\text {eff }}=\sigma_{\text {air }}\left[X_{\text {fuel }}\left(\frac{\sigma_{\text {fuel }}}{\sigma_{\text {air }}}-1\right)+1\right] .
$$

As cross-sections of hydrocarbon molecules are usually greater than those of the oxidizer constituents, $\left(\sigma_{\text {fuel }} / \sigma_{\text {air }}-1\right)$ is positive and the Rayleigh signal will increase linearly with mole fraction $X_{\text {fuel }}$.

\subsubsection{Rayleigh scattering for determination of temperature}

Under conditions where the gas composition is not precisely known, as is the case for reactive combustion environments, mole fractions $X_{i}$ might be determined from the species sensitive Raman signal, which in turn allows to determine the effective cross-section $\sigma_{\text {eff }}$ and eventually temperature. Where Raman information is not available one has to deal with a number of assumptions to bypass the unknown composition of the gas. 
In premixed methane-air flame, near stoichiometric combustion, chemistry can be roughly approximated by a single step reaction

$$
\mathrm{CH}_{4}+2 \mathrm{O}_{2} \rightarrow \mathrm{CO}_{2}+2 \mathrm{H}_{2} \mathrm{O} \text {. }
$$

One can differentiate the cold reactants region and the stream of hot product and apart from inert nitrogen $\mathrm{N}_{2}$, the above reaction equation determines the local composition on either side of the flame front.

This allows a priori estimates of the effective Rayleigh cross-section $\sigma_{\text {eff }}^{\text {cold }}$ and $\sigma_{\text {eff }}^{\text {hot }}$ for the cold reactants and hot product gases respectively,

$$
\begin{aligned}
& \sigma_{\text {eff }}^{\text {cold }}=\sigma_{\mathrm{CH}_{4}} X_{\mathrm{CH}_{4}}+\sigma_{\mathrm{O}_{2}} X_{\mathrm{O}_{2}}+\sigma_{\mathrm{N}_{2}} X_{\mathrm{N}_{2}} \text { and } \\
& \sigma_{\mathrm{eff}}^{\text {hot }}=\sigma_{\mathrm{CO}_{2}} X_{\mathrm{CO}_{2}}+\sigma_{\mathrm{H}_{2} \mathrm{O}} X_{\mathrm{H}_{2} \mathrm{O}}+\sigma_{\mathrm{N}_{2}} X_{\mathrm{N}_{2}} .
\end{aligned}
$$

It turns out that the two cross-sections are almost identical and hence the scattering intensity $I_{\Omega}$ is reciprocally proportional to temperature $T$. The mean Rayleigh cross-section for reactants is $6.56 \times 10^{-28} \mathrm{~cm}^{2} / \mathrm{sr}$ whereas it is equal to $6.50 \times 10^{-28} \mathrm{~cm}^{2} / \mathrm{sr}$ for the products assuming a complete reaction and an equivalence ratio of 0.96 . Therefore, the errors for assuming constant cross-section account for less than $1 \%$.

\subsubsection{Experimental setup}

Two-dimensional Rayleigh scattering is used to determine the spatial distribution of fuel in axial and transverse planes downstream of the burner outlet under non-reacting conditions. The same technique is employed under reacting flow conditions to examine the flame behavior in an axial plane. The Rayleigh scattering signal is dominated in that case by effects of the number density gradient at the flame front and reflects the separation between cold reactants and hot products. The field of view covers approximately $25 \times 25 \mathrm{~mm}^{2}$ and is located $2 \mathrm{~mm}$ above the nozzle outlet to avoid recording reflected light from the nozzle and burner body.

A schematic outline of the experimental setup is shown in Fig. 3. A frequency-doubled pulsed Nd:YAG laser (Continuum, Surelite SLII-10) at $532 \mathrm{~nm}$ with approximately $300 \mathrm{~mJ}$ per pulse is employed. The reflection of a glass plate is directed on a laser energy meter (Ophir, Laserstar) to monitor and compensate for fluctuations in laser output energy. In axial imaging polarization of the vertically polarized laser light remains unaltered. In the case of transverse laser sheet alignment the beam passes through a $90^{\circ}$ polarization rotator in order to assure detection of the Rayleigh signal normal to the polarization plane. A laser sheet is generated via a pair of a cylindrical and spherical lenses $\left(f_{\text {cyl }}=-50 \mathrm{~mm}\right.$ and $\left.f_{\mathrm{sph}}=250 \mathrm{~mm}\right)$ to form a laser sheet covering the field of view. The detection side of the setup consists of an Intensified Charge-Coupled Device (ICCD) camera (Princeton Instruments, PI-MAX THM512x512, GenII intensifier) equipped with a $60 \mathrm{~mm}$ objective (Nikon, AF Micro Nikkor, $60 \mathrm{~mm}$, 1:2.8) coupled to a focal length doubler (Tamron-F, 7MC Tele-Converter). 


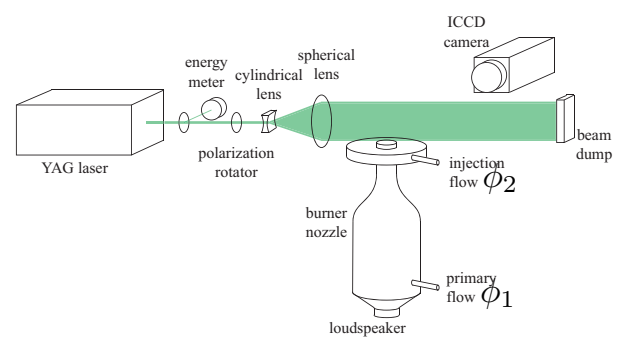

Figure 3: Schematic representation of the experimental arrangement used for Rayleigh scattering.

Timing is controlled by two pulse/delay generators (BNC, Model 555). The first triggers the laser flashlamps at $10 \mathrm{~Hz}$, as is required by the laser system, while the second is gated by the latter and releases the Q-switch and the camera on every fifth trigger pulse to allow image data read out. The ICCD camera operates in a gated mode in which the intensifier serves as an electric shutter and defines nanoseconds exposition times. The gate width is set to $25 \mathrm{~ns}$ which is largely sufficient to observe the instantaneous Rayleigh scattering response to the $\sim 10$ ns laser pulse while at the same time being much smaller than characteristic fluid and flame time scales. This permits acquisition of instantaneous images and for phase-locked data the function generator of the loudspeaker, which modulates the primary flow, serves as a master trigger for the Rayleigh scattering system.

Rayleigh scattering cross-sections at $532 \mathrm{~nm}$ are provided by Masri et al. (1996), where $\sigma_{\mathrm{CH}_{4}}=13.4 \times 10^{-28} \mathrm{~cm}^{2} / \mathrm{sr}$ for methane, $\sigma_{\mathrm{C}_{3} \mathrm{H}_{8}}=79.0 \times$ $10^{-28} \mathrm{~cm}^{2} / \mathrm{sr}$ for propane and $\sigma_{\text {air }}=5.9 \times 10^{-28} \mathrm{~cm}^{2} / \mathrm{sr}$ for air of standard composition. The cross-section of propane is almost six times stronger than that of methane and propane eventually yields significantly better signal-tonoise ratios. In fact, in this work stable flame conditions found for methane turn out to be unstable for propane combustion. In what follows, images depicting fuel distribution in non-reacting conditions have been obtained with propane, while flame dynamics refer to methane-air combustion. This approach is justified by the similarity of hydrodynamic properties of the two fuels, i.e. diffusion, stoichiometric density. The use of propane in some experiments raises also questions of the difference in density of the reactive mixture and related buoyancy effects. However estimates of the Froude number indicate that this will be small and that one may safely use propane in the place of methane.

\subsection{Hydrocarbon infrared absorption}

\subsubsection{Theoretical aspects}

The Beer-Lambert law

$$
I(x)=I_{0} \exp \left(-n \sigma_{\text {abs }} x\right)
$$

DOI: 10.1007/s00348-010-0972-3 


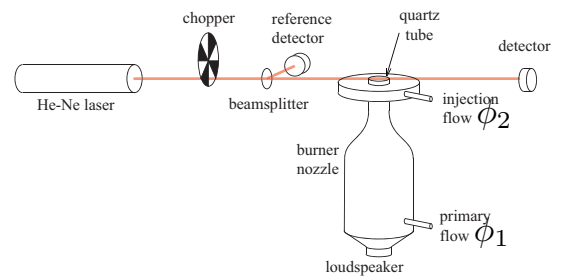

Figure 4: Schematic representation of the experimental setup used for infrared absorption spectroscopy.

relates the intensity of transmitted monochromatic light $I(x)$ to the absorption properties of the gas traversed, where $I_{0}=I(x=0), x$ is the path length and $\sigma_{\text {abs }}$ the effective absorption cross-section. Again $\sigma_{\text {abs }}$ is the weighted sum of the individual cross-sections $\sum_{i} X_{i} \sigma_{i}$ and in the case where one species' crosssection dominates the others, namely that of the hydrocarbon near $3.39 \mu \mathrm{m}$, i.e. $\sigma_{\mathrm{CH}_{4}} \gg \sigma_{i} \forall i \neq \mathrm{CH}_{4}$, the argument of equation (9) can be approximated by

$$
I(x)=I_{0} \exp \left(-n X_{\mathrm{CH}_{4}} \sigma_{\mathrm{CH}_{4}} x\right) .
$$

This can be solved for $X_{\mathrm{CH}_{4}}$ and constitutes a measure for the fuel mole fraction

$$
X_{\mathrm{CH}_{4}}=-\ln \left(\frac{I}{I_{0}}\right) \frac{k T}{\sigma_{\mathrm{CH}_{4}} p x} .
$$

\subsubsection{Experimental setup}

In the $3.39 \mu \mathrm{m}$ infrared region hydrocarbons exhibit unique absorption characteristics which can be used to make quantitative measurements of fuel concentration. The absorption cross-section of methane $\sigma_{\mathrm{CH}_{4}}$ is $21.1 \pm 3 \% \mathrm{~m}^{2} / \mathrm{mol}$ at $3.39 \mu \mathrm{m}$, temperature $T=298 \mathrm{~K}$ and atmospheric pressure $p=1 \mathrm{~atm}$ (Klingbeil et al., 2007) and absorption of other gases, such as $\mathrm{CO}, \mathrm{CO}_{2}, \mathrm{H}_{2} \mathrm{O}$ and $\mathrm{N}_{2}$ is negligible (Tomita et al., 2003). The experimental setup used in the infrared absorption experiment is depicted in Fig. 4. The transmittance of a continuous infrared Helium-Neon (He-Ne) laser at $3.39 \mu \mathrm{m}$ (Thorlabs, H339P2-1, $2.0 \mathrm{~mW}$ ) is detected by means of two infrared InSb detectors (InfraRed Associates, Inc., $\mathrm{InSb}$ ) cooled by liquid nitrogen. The laser beam is modulated via an optical chopper (Thorlabs, MC1000A) operating at $6000 \mathrm{~Hz}$ and the signal is analyzed by two lock-in amplifiers (Stanford Research Systems, SR510). The probe beam passes through a quartz tube, constituting the burner outlet duct and allowing optical access to the feed flow. While the flame is anchored on the rim of the outlet duct, the beam passes through a non-reacting environment and therefore is not subject to beam steering or scattering by soot. 


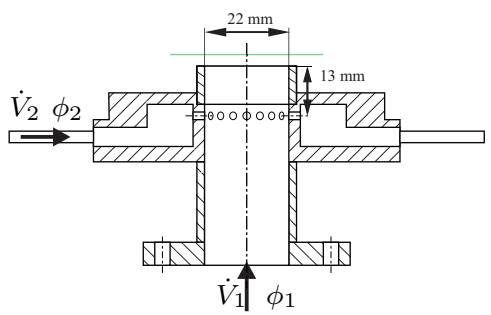

Figure 5: Sectional drawing of the injection manifold. The outlet duct is replaceable and can be substituted by a quartz tube in order to allow infrared absorption in the feed flow. Also shown is the laser sheet position for transverse fuel distribution imaging.

\section{Burner characteristics}

The burner nozzle maintains a $\sim 2 \mathrm{~kW}$ conical flame anchored on the rim of its outlet duct. To obtain gradients in the mixture fraction field the burner features an injection manifold as illustrated in Fig. 5. While the primary flow is a perfectly premixed mixture of equivalence ratio $\phi_{1}$, a secondary flow of equivalence ratio $\phi_{2}$ is injected in the form of transverse jets through orifices in the duct close to the outlet. The injection unit of the burner is removeable and the injector employed in the present study comprises either 16 or 32 holes of $1 \mathrm{~mm}$ diameter. The primary flow velocity can be acoustically modulated via a loudspeaker mounted at the bottom of the system (Fig. 4). Perturbations in mixture composition are obtained by inducing velocity modulations in the primary stream and keeping the secondary injection flow rate constant:

$$
\dot{V}_{1}={\overline{V_{1}}}_{1}+\dot{V}_{1}^{\prime} \quad \text { and } \quad \dot{V}_{2}=\text { const } .
$$

where $\dot{V}_{1}$ and $\dot{V}_{2}$ designate the volume flow rates of the primary and secondary injection flows respectively. It is of interest to estimate the fluctuation amplitudes of the composition perturbation. By assuming a sinusoidal velocity perturbation $\dot{V}_{1}={\overline{V_{1}}}_{1}+\dot{V}_{1}^{\prime} \cos (\omega t)$, an injection flow rate $\dot{V}_{2} \ll \dot{V}_{1}$ and perfect mixing a first order estimation of the resulting perturbation of the overall equivalence ratio $\phi=\bar{\phi}+\phi^{\prime}$ is readily found to be:

$$
\phi^{\prime} \approx \frac{1+\mathrm{r}_{\mathrm{st}} \phi_{1}}{1+\mathrm{r}_{\mathrm{st}} \phi_{2}}\left(\phi_{2}-\phi_{1}\right) \frac{\dot{V}_{2}}{\dot{\bar{V}}_{1}} \frac{\dot{V}_{1}^{\prime}}{\dot{V}_{1}}
$$

where $r_{s t}$ is the stoichiometric fuel-to-air ratio. It is favorable to impose small overall flow rate perturbations $\dot{V}^{\prime} / \overline{\dot{V}}$ in order to decouple the effects due to velocity and mixture perturbation on the flame. When the primary flow is modulated the equivalence ratio oscillations $\phi^{\prime}$ is proportional to $\dot{V}_{1}^{\prime} / \overline{\dot{V}_{1}}$ and will be bound to overall flow oscillations, as $\dot{V}^{\prime} / \overline{\dot{V}} \approx \dot{V}_{1}^{\prime} / \bar{V}_{1}$. 

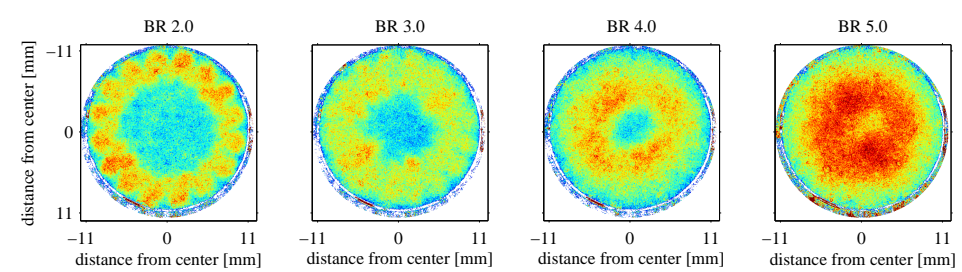

(a) 16 hole injector

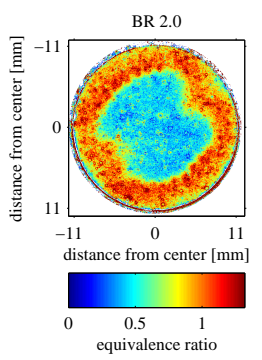

BR 3.0
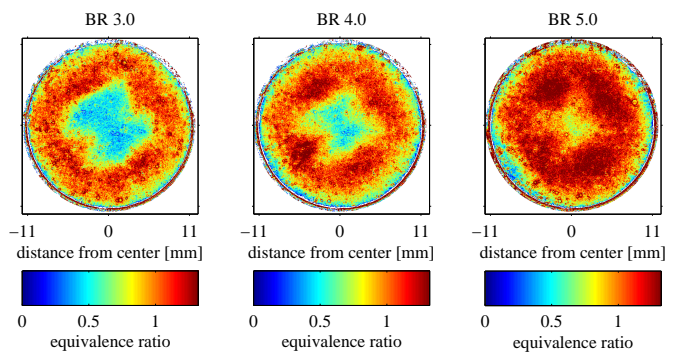

(b) 32 hole injector

Figure 6: Horizontal injection distribution for different blowing ratios (BR) for different injectors, (a) 16 injection holes $1 \mathrm{~mm}$ in diameter and (b) 32 injection holes $1 \mathrm{~mm}$ in diameter. The angular asymmetry apparent in the bottom images corresponds to the locations where the feed line is connected to the injector manifold in the upper right and lower left corners. The images displayed in the lower row are perturbed by the presence of a significant amount of particles which could not be completely eliminated by post-processing. The primary and injection flow equivalence ratio are $\phi_{1}=0.55$ and $\phi_{2}=3.40$ respectively. 
Another important consideration concerns the penetration distance of the secondary jets. The distance is governed by the blowing ratio BR (Hasselbrink and Mungal, 2001):

$$
\mathrm{BR}=\left[\frac{\rho_{2} v_{2}^{2}}{\rho_{1} v_{1}^{2}}\right]^{1 / 2} .
$$

which appears to be the square root of the momentum flux ratio used in many studies of coaxial injection. The penetration distance of the injected jets is characterized by this parameter a point confirmed by images displayed in Fig. 6 which show the equivalence ratio distributions generated by two different injectors, comprising 16 and 32 injection holes $1 \mathrm{~mm}$ in diameter. These images show distributions measured in a transverse plane under non-reactive conditions. The laser sheet is approximately $3 \mathrm{~mm}$ above the burner exit and $16 \mathrm{~mm}$ from the injector holes (cf. Fig. 5). Here the primary and the injection flow rate are constant and the primary flow consists in a lean propane-air mixture $\left(\phi_{1}=0.55\right)$ whereas the injection flow is rich $\left(\phi_{2}=3.40\right)$ to enhance contrast. It can be seen that the penetration distances are identical in the two cases if the blowing ratio is conserved. The angular non-uniformities result from the connection point of the feed line. It is also apparent that diffusion effects do not dominate over convection of the injected jets. In order to maintain the mixture composition gradient until it encounters the flame front rapid diffusion is undesirable in this context.

In the steady state, the penetration distance is proportional to the blowing ratio. Assuming that the unsteady flow evolves in a quasi-steady fashion one deduces that perturbations of the velocity $v_{1}$ will be reflected in fluctuations in the penetration length. The modulation of the primary flow does not only produce a temporal equivalence ratio oscillation (as described above) but it also generates a spatial stratification as a result of varying penetration distance. This can be seen by considering a primary flow modulation of the form $v_{1}=$ $\bar{v}_{1}+v_{1}^{\prime} \cos (\omega t)$, a constant injection flow rate and equal densities $\rho_{1}$ and $\rho_{2}$, which is generally the case for near-stoichiometric gaseous fuel-air mixtures. Then the time varying blowing ratio is

$$
\mathrm{BR}=\overline{\mathrm{BR}} \frac{1}{1+\left(v_{1}^{\prime} / \bar{v}_{1}\right) \cos (\omega t)},
$$

where $\overline{\mathrm{BR}}=\bar{v}_{2} / \bar{v}_{1}$. It can be seen that penetration is governed by the relative velocity fluctuation $v_{1}^{\prime} / \bar{v}_{1}$ and maximal penetration $\mathrm{BR}_{\max }=\overline{\mathrm{BR}}\left(1-v_{1}^{\prime} / \bar{v}_{1}\right)^{-1}$ is reached for $\cos (\omega t)=-1$.

\section{Results}

The following results were obtained in systematic experiments on the effects of equivalence ratio non-uniformities on the dynamics of a weakly turbulent flame. The first section describes the domain of operation chosen for this study. The second section presents Rayleigh scattering data under non-reacting and 
reacting conditions. The last section discusses measurements from absorption spectroscopy.

\subsection{Injection implications and turbulent flame regime}

One difficult aspect of the present investigation is to get the required stratification in the flow. The objective is to obtain a sufficiently uniform distribution of equivalence ratio in each transverse plane. It is found that this is achieved if the secondary jet penetration in the main flow is sufficient. It is shown in the previous section that this requires relatively large blowing ratios BR. This has the consequence that the flow cannot be maintained laminar. The Reynolds number of the flow $\operatorname{Re}=D \bar{v}_{1} / \nu$ is of the order of 2500 and without injection the regime would be laminar in the exhaust duct. However, the flow becomes turbulent when the secondary flow is set in operation. One may consider for example that the injected jets confine the primary flow area, thus forming an aerodynamic nozzle which increases the flow velocity and induces turbulent fluctuations. This implies that the burner system will operate in a turbulent combustion regime rather than under the laminar flame conditions referred to in section 1. Fig. 7 (a) and (b) display photographs of the laminar flame obtained in the absence of secondary injection and of the turbulent flame obtained when secondary injection is switched on at a blowing ratio $\mathrm{BR}=3$. While flow rates and equivalence ratios for both flames are identical $(\phi=0.96)$ and the secondary injection has the same composition as the main stream the flames are quite different. The corresponding mean flame positions, revealed by Rayleigh scattering, are displayed in Fig. 8, while single shots are shown in Fig. 9. One can clearly see on the turbulent cases that flame front's shape is typical of wrinkled flames regime. The power spectral density of the flow configuration with injection is shown in Fig. 10, which indicates that turbulence is not fully developed with an inertial subrange covering a few hundred hertz. This spectra was obtained using hot-wire anemometry with a measurement point located in the center of the burner's exit and using only air in both feed and secondary flow.

General flow conditions, namely average equivalence ratio $\bar{\phi}=0.96$, bulk velocity $v_{b}=1.96 \mathrm{~m} / \mathrm{s}$ and blowing ratio $\mathrm{BR}=3$, were imposed in all experiments presented in what follows. The turbulence fluctuation $v /$ in the injectionperturbed flow was found to amount to $0.10 \mathrm{~m} / \mathrm{s}$, corresponding to $5 \%$ of the mean flow velocity. Velocities were measured at the center of the burner outlet and at an off-axis position for different excitation frequencies using a hot-wire anemometer. It turns out that the turbulence intensity v ranges from 0.090 .10 $\mathrm{m} / \mathrm{s}$ for all conditions, whereas the acoustic flow speed variations differ depending on whether the transversal injection jet strikes in the measurement region.

\subsection{Dynamics of flame subject to equivalence ratio non- uniformities}

It is natural to first examine axial distributions of the injected flow under nonreacting conditions, displayed in Fig. 11. Four consecutive phase angles $\varphi$ in 


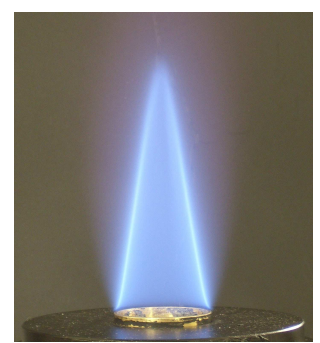

(a) laminar flame

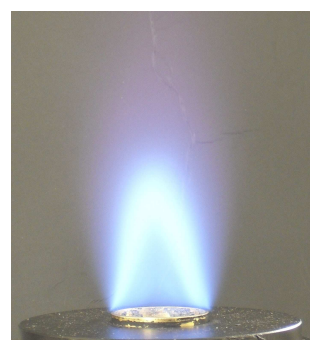

(b) turbulent flame

Figure 7: Long time exposure of (a) laminar and (b) turbulent flames. Turbulence is generated by the secondary injection flow. In case (a) there is no secondary injection. In case (b) the injected flow is on with a blowing ratio $\mathrm{BR}=3$. Both flows are not modulated and have homogenous equivalence ratios of $\phi=0.96$. Exposure time of $1 \mathrm{~s}$.

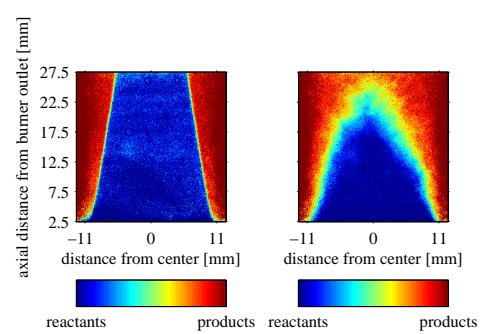

Figure 8: Mean flame front of the laminar flame on the left and the turbulent flame on the right. Conditions are $\phi=0.96$ in both cases and the turbulent flame is subject to an injection flow with $\mathrm{BR}=3$.
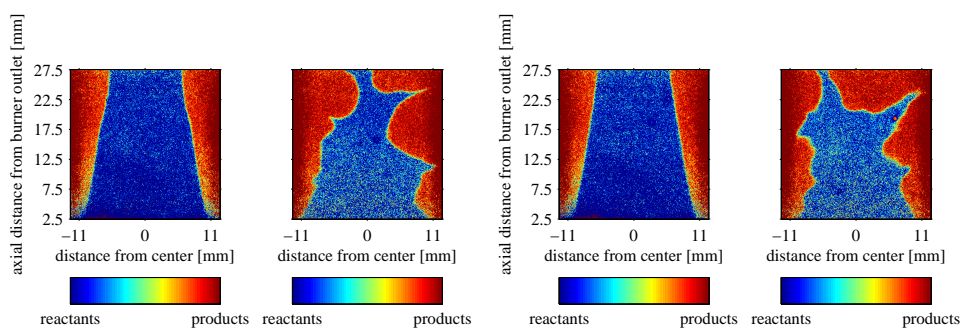

Figure 9: Typical single shots of the flame front. The laminar flame is on the left and the injection-induced turbulent flame on the right. Same conditions as in Fig. 8.

Accepted in Experiments in Fluids

DOI: $10.1007 / \mathrm{s} 00348-010-0972-3$ 


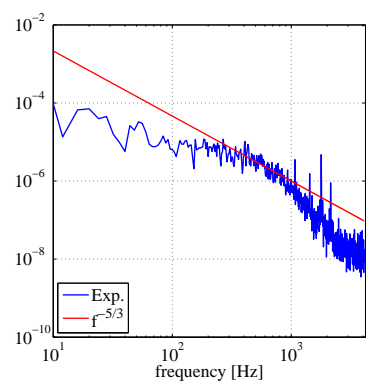

Figure 10: Power spectral density (PSD) of the velocity signal measured in the duct exhaust section obtained using hot-wire anemometry. Secondary injection is switched on with $\mathrm{BR}=3$. The red graph represents the $f^{-5 / 3}$ spectral decay.

a cycle show the evolution of the flow exhausted by the system modulated at a frequency $f=200 \mathrm{~Hz}$. The signal to noise ratio allows a relatively limited quantification of the actual fuel concentration, but the injected flow distribution is revealed giving a good representation of the convective and diffusive effects in the flow.

Two layers of the injected flow are clearly visible and appear in the field of view. These are convected downstream and under reactive conditions these layers will impinge on the flame front. The nominal convective wavelength of the fuel-rich layers $\lambda=\bar{v} / f$ is slightly smaller than the effective wavelength due to a slight acceleration in the nozzle associated with the injection stream. Even for a mean value of the blowing ratio of 3 , fuel reaches the axis and forms horizontal stripes during part of the cycle. This is in contrast with the steady operation, illustrated in Fig. 6 where fuel remains in the annular layer adjacent to the wall. This is because under unsteady operation, the blowing ratio takes large values allowing fuel penetration and formation of the desired layers.

The resulting flame contours are depicted in Fig. 12 for three different perturbations in mixture composition. Phases $\varphi$ correspond to those of Fig. 11 and bulk velocity is identical. The equivalence ratio of the secondary flow $\phi_{2}$ ranges from 0 to 7.84 , while the mean overall equivalence ratio $\phi=0.96$ is constant. It is interesting to consider this large interval of injection equivalence ratios $\phi_{2}$ ranging from pure air to a very rich mixture. The flame contour displayed in the middle row represents the homogenous reference condition where the equivalence ratios of the primary and the injection flow are equal. Characteristics of this contour are dominated by the flow velocity perturbations since the chemical composition of the fresh stream is constant and the equivalence ratio is fixed.

It appears clearly that the flame front dynamics is dominated by velocity perturbations imposed by the loudspeaker and the injection jets, and that effects related to equivalence ratio perturbations are less pronounced. Even in the absence of any secondary injection, a radial velocity component is generated when the axial velocity is modulated just to satisfy the conservation of mass equations. When the injected flow is operating there is an additional perturbation 

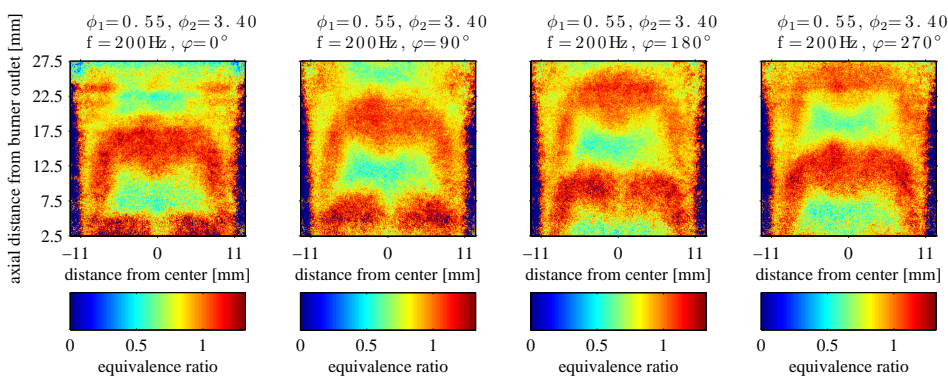

Figure 11: Equivalence ratio distributions in nonreactive conditions for four phase angles $\varphi$. The excitation frequency is $f=200 \mathrm{~Hz}$, the equivalence ratios of the primary and the injected flow are $\phi_{1}=3.40$ and $\phi_{2}=0.55$ respectively and the blowing ratio is $\overline{\mathrm{BR}}=3$. Propane is used in this experiment used to augment the signal to noise ratio.

in the radial velocity component. These two types of velocity perturbations are responsible for the inward wrinkling of the flame and for the major part of the fluctuation in flame surface area. This is readily illustrated by superimposing the mean flame front images and the injected layers (Fig. 13). It is important to point out that in this context the pattern must not be interpreted in terms of mixture composition distribution but has to be seen as a flow pattern showing the injection jet streaklines. In other words, the red colored layers correspond to a region of small fuel concentration in the case $\phi_{2}=0$ and to a rich zone in the case $\phi_{2}=7.84$, while the flame contour remains nearly unchanged. However, some modifications are perceptible which are most likely linked to the mixture inhomogeneities. For example, by comparing the first phase $\varphi=0^{\circ}$ of the three conditions in Fig. 12 one perceives an increasing curling effect on the top cap of the flame. Although we have observed that the equivalence ratio non-uniformities have a weaker impact on the flame surface area they might nevertheless produce a modulation in heat release $q^{\prime}$ and couple to pressure perturbations as was described in the introductory section. This effect could not be investigated experimentally in the context of this work.

So far the effective equivalence ratio perturbation amplitude impinging on the flame has not been precisely quantified. This will be done in the following section by making use of the hydrocarbon absorption technique.

\subsection{Temporal evolution of the mixture fluctuations}

The temporal equivalence ratio fluctuations are revealed by absorption experiments. The phase averaged signal is shown in Fig. 14. The flow modulation frequency $f=200 \mathrm{~Hz}$ is clearly discernable as are the varying amplitudes for the different flow configurations. In the homogenous condition when the two equivalence ratios $\phi_{1}=\phi_{2}=0.96$ are equal (diamonds - green line) the fluctuation 

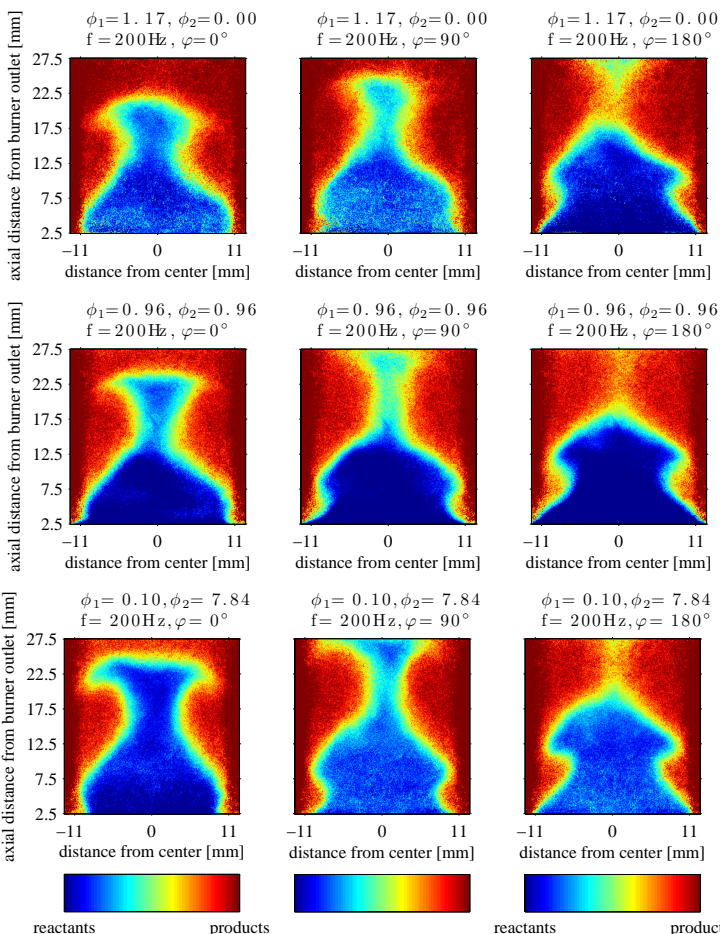

$\phi_{1}=1.17, \phi_{2}=0.00$

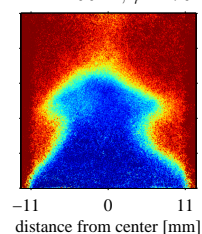

$\phi_{1}=0.96, \phi_{2}=0.96$
$\mathrm{f}=200 \mathrm{~Hz}, \varphi=90^{\circ}$

$\phi_{1}=0.96, \phi_{2}=0.96$
$\mathrm{f}=200 \mathrm{~Hz}, \varphi=180^{\circ}$

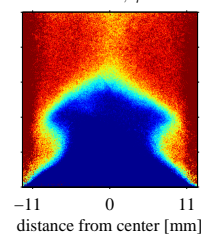

$\phi_{1}=0.96, \phi_{2}=0.96$
$\mathrm{f}=200 \mathrm{~Hz}, \varphi=270^{\circ}$
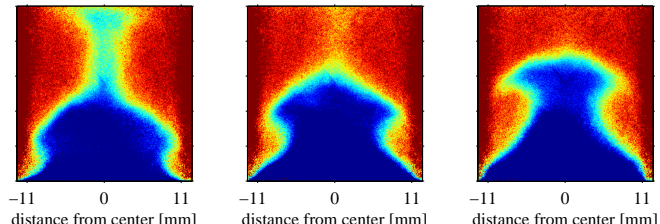

$\phi_{1}=0.10, \phi_{2}=7.84$
$\mathrm{f}=200 \mathrm{~Hz}, \varphi=90^{\circ}$

$\phi_{1}=0.10, \phi_{2}=7.84$

$\mathrm{f}=200 \mathrm{~Hz}, \varphi=18$

$\phi_{1}=0.10, \phi_{2}=7.84$

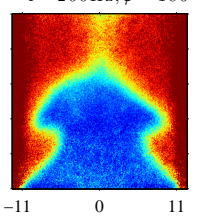

$\mathrm{f}=200 \mathrm{~Hz}, \varphi=27$
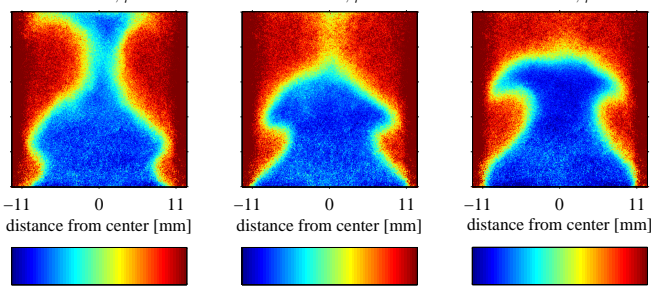

Figure 12: Mean flame front for a modulation at $200 \mathrm{~Hz}$ and an average equivalence ratio $\phi=0.96$. Top row : $\phi_{1}=1.17, \phi_{2}=0.00$. Middle row : $\phi_{1}=0.96$, $\phi_{2}=0.96$. Bottom row : $\phi_{1}=0.10, \phi_{2}=7.84$.
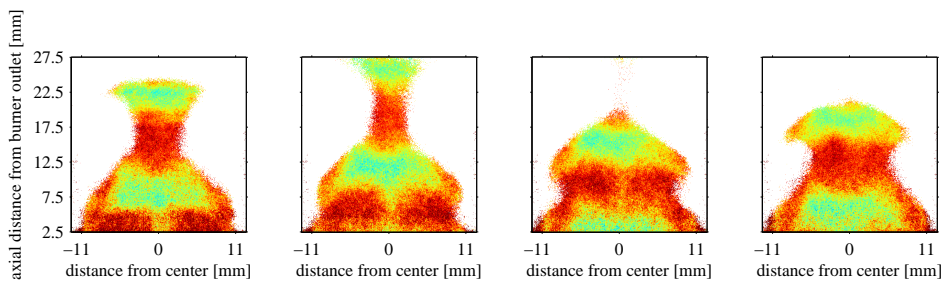

Figure 13: Superimposed mean flame front and distribution of the injection jets for a $200 \mathrm{~Hz}$ modulation.

Accepted in Experiments in Fluids

DOI: $10.1007 / \mathrm{s} 00348-010-0972-3$ 
amplitude vanishes. In the other conditions a significant fluctuation of the fuel concentration is observed. The shift in mean equivalence ratio is most likely explained by non-uniform transverse fuel distributions generated by the injection system and their integration over the line-of-sight. This operation biases the overall mixture composition measurement and is most important when there is a large difference in equivalence ratio between the primary and the injection flow.

It is possible to use these data to estimate the fluctuation scale of the mixture composition perturbations. The injected volumetric flow rate is not negligible $\left(\dot{V}_{2} / \bar{V}_{1}=0.23\right)$ and one has to use a nonlinear version of equation (13). The relative flow rate oscillation is $\dot{V}_{1}^{\prime} / \overline{\dot{V}_{1}}=0.18 \pm 0.02$. Because this value can not be precisely known, there is an uncertainty in the theoretical estimate. The measured and estimated fluctuation amplitudes $\phi^{\prime}$ gathered in Table 1 essentially agree. This shows that uncertainties in the measurements due to the absorption technique are relatively limited and that $4 \%$ may be used as an upper bound of those, as obtained in the calibration procedure.

\begin{tabular}{|c|c|l|c|}
\hline$\phi_{1}$ & $\phi_{2}$ & \multicolumn{1}{|c|}{$\phi_{\text {theo }}^{\prime}$} & $\phi_{\exp }^{\prime}$ \\
\hline 1.17 & 0.00 & $0.04 \pm 0.01$ & $0.04 \pm 0.01$ \\
0.96 & 0.96 & 0.00 & $0.00 \pm 0.01$ \\
0.55 & 3.40 & $0.07 \pm 0.01$ & $0.06 \pm 0.01$ \\
0.10 & 7.84 & $0.14 \pm 0.02$ & $0.13 \pm 0.01$ \\
\hline
\end{tabular}

Table 1: Comparison of the calculated equivalence ratio fluctuation amplitude $\phi_{\text {theo }}^{\prime}$ and the equivalence ratio fluctuation amplitude $\phi_{\exp }^{\prime}$ measured by absorption spectroscopy.

In conclusion, it is reasonable to say that the scale of the equivalence ratio perturbations in the present experiments amount to $\sim 0.1$ and have the order of magnitude expected initially. This order of magnitude is of interest for the discussion outlined in the following section.

\section{$5 \quad$ Premixed flame dynamics}

To discuss results obtained in the previous section, it is convenient to examine the dynamics of premixed flames submitted to incident velocity and equivalence ratio perturbations. This analysis is only intended to provide estimates of orders of magnitude of the various terms influencing the flame interaction with incident disturbances. This is based on an ensemble average G-equation as exemplified by Peters (2000). Stretch effects are not very large in the present experiments and are therefore neglected. 


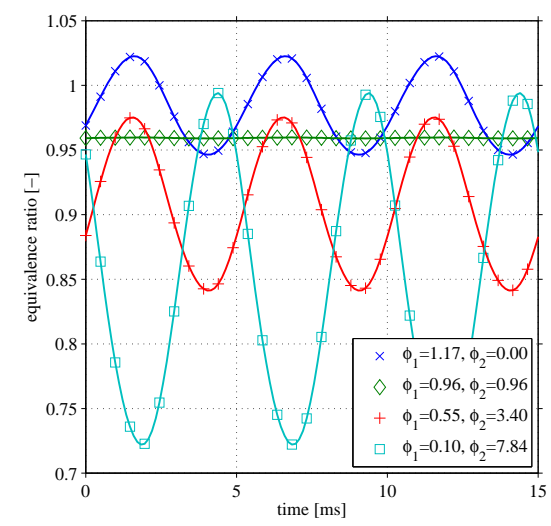

Figure 14: Equivalence ratio fluctuation revealed by infrared absorption at the outlet burner. The phase-averaged equivalence ratio perturbation and the average values are shown. Measurements are presented for different primary $\phi_{1}$ and injected mixtures $\phi_{2}$ while the global equivalence ratio $\phi$ remains constant. The primary flow is modulated at $200 \mathrm{~Hz}$ and there is no phase correlation between the signals.

\subsection{Evolution equation of premixed flame front}

The flame front in premixed flow conditions can be treated as a thin interface separating hot products from the fresh mixture. Its evolution can be tracked by means of a level set approach where a scalar field $G(t, \boldsymbol{x})$ characterizes time dependent iso-surfaces. One of the levels $G_{0}$ defines the flame surface $\left\{\boldsymbol{x}_{f} \mid G\left(t, \boldsymbol{x}_{f}\right)=G_{0}\right\}$. One may consider for example that the flame corresponds to $G_{0}=0$ and that $G<0$ represents the cold reactants whereas $G>0$ pertains to the hot products. The dynamics of the flame front is given by

$$
\frac{\partial G(t, \boldsymbol{x})}{\partial t}+\boldsymbol{v} \cdot \nabla G(t, \boldsymbol{x})=s_{D}|\nabla G(t, \boldsymbol{x})|,
$$

commonly referred to as the G-equation. It features a convective term depending on the underlying flow velocity $\boldsymbol{v}$ and a displacement term relative to the fluid motion. The flame displacement is in the direction normal to the flame front given by

$$
\boldsymbol{n}=-\nabla G(t, \boldsymbol{x}) /|\nabla G(t, \boldsymbol{x})| .
$$

In the laminar case $s_{D}$ is the laminar burning velocity $s_{L}$. In the turbulent case equation (16) can be ensemble averaged and it is then replaced by

$$
\frac{\partial \bar{G}(t, \boldsymbol{x})}{\partial t}+\overline{\boldsymbol{v}} \cdot \nabla \bar{G}(t, \boldsymbol{x})=s_{T}|\nabla \bar{G}(t, \boldsymbol{x})|,
$$

where $s_{D}$ is now replaced by a turbulent burning velocity $s_{T}$ and $\bar{G}$ is the ensemble average of $G$. This is only used as a model equation. 


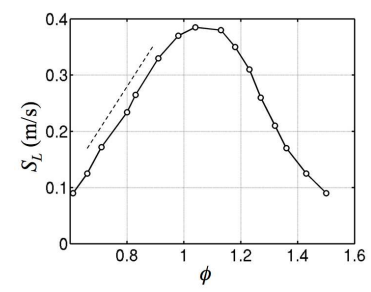

Figure 15: Laminar burning velocity $s_{L}$ of a methane-air flame at atmospheric pressure as a function of equivalence ratio $\phi$. (illustration adapted from Birbaud et al. (2008).)

The previous equations may now be used to consider the laminar case before dealing with the turbulent situation.

\subsection{Laminar conical flames}

Studies of flame dynamics have been carried out in various geometries. Effects of velocity perturbations on the flame motion are investigated for example by Schuller et al. (2002) who show that the problem can be suitably analyzed by means of the G-equation (16). The perturbation is introduced in form of a convective velocity oscillation of amplitude $\hat{v}$ around the steady axial mean flow velocity $\bar{v}$,

$$
v(t, \boldsymbol{x})=\bar{v}+\hat{v} \exp (i k x-i \omega t),
$$

where the convective wavenumber $k=\omega / \bar{v}$.

Another standard problem is that of an equivalence ratio perturbation of the generic form

$$
\phi=\bar{\phi}+\hat{\phi} \exp (i(k x-\omega t+\psi))
$$

This perturbation influences the laminar burning velocity $s_{L}$, as illustrated in Fig. 15. The angle $\psi$ represents the phase shift between velocity and equivalence ratio perturbations.

In lean combustion and for small perturbations the laminar burning velocity $s_{L}$ varies linearly with the equivalence ratio disturbances, as illustrated by the dashed line in Fig. 15, hence

$$
s_{L}(\phi)=s_{L}(\bar{\phi})+\left.\frac{\partial s_{L}}{\partial \phi}\right|_{\bar{\phi}} \phi^{\prime} .
$$

To analyze the interaction between the flame and the incident perturbations it is convenient to use the method introduced by Schuller et al. (2003). The laboratory frame $\boldsymbol{x}=(x, y)$ is replaced by a reference frame attached to the steady state flame front coordinates and the new coordinates are given by

$$
\boldsymbol{X}=(X, Y)=(x \sin \alpha+y \cos \alpha,-x \cos \alpha+y \sin \alpha)
$$

DOI: 10.1007/s00348-010-0972-3 
The half-angle of a conical stationary flame front $\alpha$ is determined by the standard relation $\sin \alpha=s_{L}(\bar{\phi}) / \bar{v}$ and gives the angle between the two coordinate systems. The scalar field $G(t, \boldsymbol{X})$ is then expressed in terms of the normal flame displacement $\xi(X, t)$ so that $G(t, \boldsymbol{X})=Y-\xi(t, X)$. A first order analysis then yields a dynamical equation for the normal flame displacement:

$$
\frac{\partial \xi(t, X)}{\partial t}+\bar{U} \frac{\partial \xi(t, X)}{\partial X}=V^{\prime}-s_{L}^{\prime}
$$

The right hand side features a term $V^{\prime}$ corresponding to the relative velocity perturbation projected on the normal to the steady flame and a term related to the burning velocity perturbations. It can be seen that the flame front is modulated in the same way by velocity perturbations $V^{\prime}$ frame and by the burning velocity disturbance $s_{L}^{\prime}$. In terms of the sinusoidal perturbations (18) and (19) the right hand side forcing function is readily cast in the form

$$
s_{L}\left[\frac{\hat{v}}{\bar{v}}-\left.\frac{1}{s_{L}} \frac{\partial s_{L}}{\partial \phi}\right|_{\bar{\phi}} \hat{\phi} \exp (i \psi)\right] \exp (i(k X \cos \alpha-\omega t))
$$

where it is assumed that the transverse velocity perturbations in the laboratory frame are small compared to the axial velocity perturbations $u^{\prime} \ll v^{\prime}$. It is interesting to first note that the flame displacement is greatest for the largest values of $s_{L}$. It is then possible to make some estimates of the order of magnitude of the two terms inside the brackets. In the laminar regime the velocity perturbation $\hat{v} / \bar{v}$ can be of the order $O(\hat{v} / \bar{v})=10^{-2} \div 10^{-1}$. For methane flames the factor $\left(1 / s_{L}\right)\left(\partial s_{L} / \partial \phi\right)$ in the second term is approximately $\sim 1.3$ for a mean equivalence ratio around $\bar{\phi}=0.96$ and increases with decreasing equivalence ratio. Evidently, near stoichiometry the gradient vanishes and small mixture perturbations will have virtually no effect. On the lean combustion side the whole term will be of the order of the equivalence ratio oscillation $O\left(\phi^{\prime}\right)$ and will be of the order of $10^{-2}$ for natural fluctuations, but for control mechanisms it might be feasible to impose a $\phi^{\prime}$ which may reach up to $10^{-1}$. In this case both terms are of the same order of magnitude.

\subsection{Weakly turbulent flames}

To define the burning velocity in a weakly turbulent situations like that considered in the present experiments one may use a simple model proposed by Damköhler which consists in assuming that the turbulent flame is a locally wrinkled laminar flame. As supported by experimental results shown in Fig. 16 a linear model may be used

$$
\frac{s_{T}}{s_{L}}=1+C \frac{v_{t}^{\prime}}{s_{L}}
$$

where $C$ is an experimental correlation factor and the root mean square turbulent velocity fluctuation $v_{t}^{\prime}$ is related to the turbulent kinetic energy $k=$ 


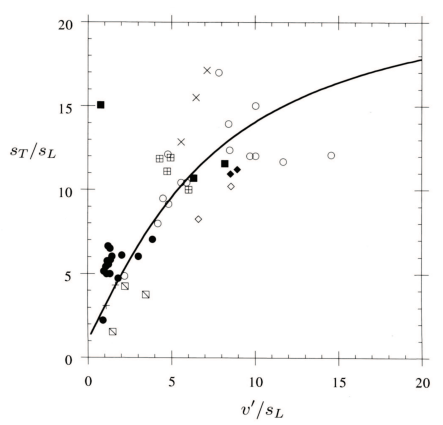

Figure 16: Turbulent flame speed as a function of the turbulence intensity $v^{\prime}$. Experimental results correspond to Reynolds numbers ranging from $R e_{t}=500$ - 750 and the calculated burning velocity model is for $R e_{t}=625$ (originating from Peters (2000))

$(1 / 2) \overline{\boldsymbol{v}_{t}^{\prime} \cdot \boldsymbol{v}_{t}^{\prime}}$ through $v_{t}^{\prime}=[(2 / 3) k]^{1 / 2}$. This model equation will be used to interpret some of the findings of the present study. This is an admittedly simplified description of turbulent combustion but is convenient for the following discussion. It is clear that it cannot represent the complexity of turbulent combustion in more general situations (see for example Peters (2000)).

\subsection{Discussion of experimental results}

At this point it is worth discussing some of the issues raised by the previous experiments. It is first interesting to examine the effects of turbulence on the flame. This can be done by measuring the half-angle $\alpha$ with respect to the vertical direction of the laminar and the turbulent flame and can be used to measure the burning velocity. The half angle corresponding to the laminar case is $\alpha_{L}=9^{\circ}$. This angle is augmented in the turbulent case and takes values $\alpha_{T}=16^{\circ} \div 21^{\circ}$ (these values are deduced from Fig. 8). Hence, the ratio of the turbulent and laminar flame burning velocities can be estimated to be $s_{T} / s_{L}=\sin \alpha_{T} / \sin \alpha_{L} \approx 2.1$ and the factor $C$ appearing in the turbulent burning velocity for weak turbulence (equation (23)) is readily calculated to be

$$
C=\frac{s_{T} / s_{L}-1}{v^{\prime} / s_{L}}=3.3 .
$$

This value is consistent with Fig. 16. One can then reconsider the perturbation function (22) appearing in the linearized G-equation, where the effects of equivalence ratio variations in the turbulent regime is given by the term

$$
\frac{1}{s_{T}} \frac{\partial s_{T}}{\partial \phi} .
$$

Now, the turbulent burning velocity in weak turbulence $s_{T}$ depends on the equivalence ratio like the laminar burning velocity, so that $\left(\partial s_{T} / \partial \phi\right) \simeq\left(\partial s_{L} / \partial \phi\right)$ and 
as a consequence

$$
\frac{1}{s_{T}} \frac{\partial s_{T}}{\partial \phi}=\frac{s_{L}}{s_{T}} \frac{1}{s_{L}} \frac{\partial s_{L}}{\partial \phi}
$$

Since $s_{L} / s_{T} \approx 0.5$ one finds that the relative change in burning velocity with respect to equivalence ratio variations is about half of that corresponding to the laminar case

$$
\frac{1}{s_{T}} \frac{\partial s_{T}}{\partial \phi}=0.5 \frac{1}{s_{L}} \frac{\partial s_{L}}{\partial \phi}
$$

In general, the effect of equivalence ratio perturbations on the turbulent burning velocity will be less pronounced than in the laminar case. As is suggested by right hand side forcing function of the G-equation (22) the influence of velocity and equivalence ratio fluctuations is governed by the two factors

$$
\frac{\hat{v}}{\bar{v}} \quad \text { and } \quad \frac{1}{s_{T}} \frac{\partial s_{T}}{\partial \phi} \phi^{\prime}
$$

In the present experiments the relative velocity fluctuation imposed by the driver unit is of the order 0.2 and the equivalence ratio perturbation term amount to 0.07. This may explain why effects of equivalence ratio perturbations are not as important as one would have expected initially, especially in comparison with those corresponding to laminar conditions (cf Fig. 2).

\section{Conclusion and perspective}

The present work was carried out to investigate the influence of equivalence ratio perturbations on the dynamics of premixed flames. Two laser-diagnostic methods, based on Rayleigh scattering and hydrocarbon infrared absorption respectively, have been setup. They were employed to analyze the flow originating from a novel burner designed to induce equivalence ratio fluctuations. The experimental arrangement is used to study a weakly turbulent flame subject to mixture composition inhomogeneities.

The data obtained from systematic experiments indicate that it is possible to find conditions in which the equivalence ratio is distributed in layers and convected by the mean flow. This is obtained by making use of a secondary injection manifold which is submitted to acoustic modulations of the primary flow. The blowing ratio must be about three to obtain well defined layers of mixture composition. By suitably adjusting the primary and secondary flow equivalence ratios it was possible to obtain perturbation amplitudes $\phi^{\prime}$ of the order of $\sim 0.1$ around a mean equivalence ratio $\phi=0.96$. Because the blowing ratio was relatively high, it was found that the system operates in a weakly turbulent regime where the flame front is wrinkled by the turbulent fluctuations in the flow. The interaction of this flame with different levels of equivalence ratio disturbances is then documented. The analysis of the mean flame geometry at different phases during a cycle indicates that the flame shape is mainly 
determined by the velocity perturbations imparted to the main stream and induced by this modulation. The equivalence ratio perturbations have a weaker but discernable effect.

These findings are interpreted by making use of a level-set (G-equation) method to describe the flame front dynamics. The model is adapted to weakly turbulent combustion and suggests that in this regime equivalence ratio perturbations will be of a lesser importance (by a factor of $s_{L} / s_{T}<1$ ) than what can be expected under laminar conditions. The modeling approach supports the idea that the flame shape is mainly governed by velocity perturbations which dominate over effects induced by equivalence ratio perturbations.

Results presented can already serve to guide the numerical modeling of flames submitted to combined velocity and equivalence ratio disturbances. It would be of interest to complement the experimental data by determining the velocity field by means of a particle imaging velocimetry. This will provide the required information to carry out a numerical simulation based on the Gequation model.

\section{Acknowledgements}

This article is dedicated to Jürgen Wolfrum on his seventieth birthday with our admiration for his scientific contributions to combustion science.

\section{References}

H. Ax, P. Kutne, W. Meier, K. Konig, U. Maas, A. Class, and M. Aigner. Low pressure premixed ch4/air flames with forced periodic mixture fraction oscillations: experimental approach. Applied Physics B, 94:705-714(10), 2009.

F. Biagioli, F. Güthe, and B. Schuermans. Combustion dynamics linked to flame behaviour in a partially premixed swirled industrial burner. Experimental Thermal and Fluid Science, 32(7):1344-1353, 72008.

A.L. Birbaud. Dynamique d'interactions sources des instabilités de combustion. PhD thesis, Ecole Centrale Paris, 2006.

A.L. Birbaud, S. Ducruix, D. Durox, and S. Candel. The nonlinear response of inverted $\mathrm{v}$ flames to equivalence ratio nonuniformities. Combustion and Flame, 154(3):356 - 367, 2008.

S. Candel. Combustion dynamics and control: Progress and challenges. Proceedings of the Combustion Institute, 29(1):1 - 28, 2002.

J.H. Cho and T. Lieuwen. Laminar premixed flame response to equivalence ratio oscillations. Combustion and Flame, 140(1-2):116-129, 12005.

DOI: $10.1007 / \mathrm{s} 00348-010-0972-3$ 
L. Crocco and S.L. Cheng. Theory of Combustion Instability in Liquid Propellant Rocket Motors. Butterworths Science Publication, London, 1956.

E.F. Hasselbrink and M.G. Mungal. Transverse jets and jet flames. part 1. scaling laws for strong transverse jets. Journal of Fluid Mechanics, 443(-1): $1-25,2001$.

D.M. Kang, V. Fernandez, A. Ratner, and F.E.C. Culick. Measurements of fuel mixture fraction oscillations of a turbulent jet non-premixed flame. Combustion and Flame, 156(1):214 - 220, 2009.

A.E. Klingbeil, J.B. Jeffries, and R.K. Hanson. Temperature-dependent mid-ir absorption spectra of gaseous hydrocarbons. Journal of Quantitative Spectroscopy and Radiative Transfer, 107(3):407 - 420, 2007.

J.G. Lee, K. Kim, and D.A. Santavicca. Measurement of equivalence ratio fluctuations and its effect on heat release during unstable combustion. Proceedings of the Combustion Institute, 28:415-421, 2000.

T. Lieuwen and B.T. Zinn. The role of equivalence ratio oscillations in driving combustion instabilities in low nox gas turbines. Symposium (International) on Combustion, 27(2):1809 - 1816, 1998.

A.R. Masri, R.W. Dibble, and R.S. Barlow. The structure of turbulent nonpremixed flames revealed by raman-rayleigh-lif measurements. Progress in Energy and Combustion Science, 22(4):307 - 362, 1996.

W. Meier, P. Weigand, X.R. Duan, and R. Giezendanner-Thoben. Detailed characterization of the dynamics of thermoacoustic pulsations in a lean premixed swirl flame. Combustion and Flame, 150:2-26, 2007.

R. Mongia, R.W. Dibble, and J. Lovett. Measurements of air-fuel ratio fluctuations caused by combustor driven oscillations. In $A S M E$, pages ASME Paper No. 98-GT-304, 1998.

N. Peters. Turbulent combustion. Cambridge University Press, 2000.

A.A. Putnam. Combustion-Driven Oscillations in Industry. Elsevier Publishing Company, New York, NY, USA, 1971.

A. Ratner, W. Pun, S.L. Palm, and F.E.C. Culick. Phase-resolved no planar laser-induced fluorescence of a jet flame in an acoustic chamber with excitation at frequencies $<60 \mathrm{hz}$. Proceedings of the Combustion Institute, 29(1):85-90, 2002 .

J.W.S. Rayleigh. The explanation of certain acoustical phenomena. Nature, 18: $319-321,1878$.

T. Sattelmayer. Influence of the combustor aerodynamics on combustion instabilities from equivalence ratio fluctuations. Journal of Engineering for Gas Turbines and Power, 125(1):11-19, 2003. doi: 10.1115/1.1365159.

Accepted in Experiments in Fluids 24

DOI: $10.1007 / \mathrm{s} 00348-010-0972-3$ 
T. Schuller. Mécanismes de Couplage dans les Interactions AcoutiqueCombustion. PhD thesis, Ecole Centrale Paris, 2003.

T. Schuller, S. Ducruix, D. Durox, and S. Candel. Modeling tools for the prediction of premixed flame transfer functions. Proceedings of the Combustion Institute, 29(1):107 - 113, 2002.

T. Schuller, D. Durox, and S. Candel. A unified model for the prediction of laminar flame transfer functions: comparisons between conical and v-flame dynamics. Combustion and Flame, 134(1-2):21 - 34, 2003.

E. Tomita, N. Kawahara, A. Nishiyama, and M. Shigenaga. In situ measurement of hydrocarbon fuel concentration near a spark plug in an engine cylinder using the $3.392 \mu \mathrm{m}$ infrared laser absorption method: application to an actual engine. Measurement Science and Technology, 14(8):1357-1363, 2003.

L. Zimmer and S. Tachibana. Laser induced plasma spectroscopy for local equivalence ratio measurements in an oscillating combustion environment. Proceedings of the Combustion Institute, 31(1):737-745, 2007. 